\title{
ON THE EXISTENCE OF SMALL-TIME HEAT EXPANSIONS FOR OPERATORS WITH IRREGULAR SINGULARITIES IN THE COEFFICIENTS
}

\author{
Constantine J. Callias
}

The asymptotic coefficients in the expansion of $\operatorname{Tr} e^{-t H}$ as $t \rightarrow 0+$, for $H$ a positive elliptic differential operator of even order, with smooth coefficients, on a compact manifold, are spectral invariants of $H$ given by integrals of local functions of the coefficients of $H$ [S, Gi]. They are of proven value in computations of indices [ABP, Gi] and determinants [CT, e.g.] and in inverse spectral problems [Gu, OPS]. They are also, in general, notoriously intractable. For large classes of differential operators with singular coefficients, certain expansions of a rather different type still exist but the complications seem to be compounded. If $H$ is positive elliptic of order $d$ on a manifold, with coefficients that can have singularities on submanifolds, characterized by asymptotic expansions to all orders (as in Section 1 below), the typical result is: For $\varphi$ a multiplication operator by a smooth function of bounded support,

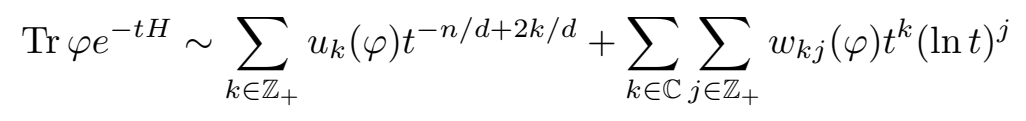

as $t \rightarrow 0$, where $u_{k}, w_{k j}$ are distributions, with the $w_{k j}$ supported on the singularities of the coefficients of $H$ and $w_{k j} \neq 0$ only for a sequence of $k$ 's with real parts increasing to $\infty$, and only finitely many $j$ 's for each $k$. Such expansions for the "distributional trace," as Seeley first referred to the left-hand side of (1), have been established by various authors for different operators with regular singularities [Ch, $\mathrm{C} 1, \mathrm{CU}, \mathrm{BrS}]$. As we explain briefly in Section 2, the mere existence of the expansion (1) is often helpful in index problems, while for inverse spectral and determinant problems laborious computations of asymptotic coefficients are normally required. It appears in fact that in all of these kinds of problems for singular operators, crucial information is often easier to extract than in the smooth case.

Received January 20, 1995. 
One would expect similar results for operators with irregular singularities, but the methods used in the regular singular case do not seem directly applicable. What we show in this note is that, seen from the right point of view, this too is simply a problem in "regular singular asymptotics." In Section 3 we give some concrete computations in dimension 1 that illustrate the main points of the proof for general irregular point-like singularities.

\section{Interior estimates and boundary behavior of functions near a corner [C3]}

As the following elementary results show, expansions of the form (1) can be established for a function $f(t)$ in $C^{\infty}(0, \infty)$ by suitable bounds on combinations of the derivatives of $f(t)$ for $t>0$. Similar ideas are included for functions of several variables. Some of the tools that we have developed for this purpose lead to a generalized notion of smoothness near a boundary with corners - "anomalous smoothness" - and are truly elementary in nature but provide very elegant solutions to many asymptotic problems, as in Proposition 2 below.

An expansion of the type we are after is characterized by a multiplicity function or asymptotic character, by which we mean an $S: \mathbb{C} \rightarrow \mathbb{Z}_{+}$such that $\sum_{\Re z<k} S(z)<\infty$ for each $k \in \mathbb{R}$. Given such an $S$, define the degree of $S$ as $m=m(S)=\sum_{z \in \mathbb{C}} S(z)$. For $f:(0, \infty) \rightarrow \mathbb{C}$ we say $f \in \Gamma^{S}(0, \infty)$ if $f \in C^{m}(0, \infty)$ and $\partial_{t}^{s} f(t)=\sum_{\Re z \leq \Re k} \sum_{0 \leq j<S(z)} f_{z j} \partial_{t}^{s}\left[t^{z}(\ln t)^{j}\right]+O\left(t^{k+\delta_{k}-s}\right)$ as $t \rightarrow 0+$, for all $k \in \mathbb{C}$ with $S(k)>0$, all integers $s$ with $0 \leq s \leq m$ and some $\delta_{k}>0$. Let $\Gamma^{\infty}(0, \infty)=\bigcup_{S, m(S)=\infty} \Gamma^{S}(0, \infty)$. If $S$ is an asymptotic character and $z \in \mathbb{C}$, let $P_{z}[S]$ be the differential operator $\prod_{\substack{\Re z^{\prime} \leq \Re z \\ z^{\prime} \neq z}}\left(x \partial_{x}-z^{\prime}\right)^{S\left(z^{\prime}\right)}$ on $C_{o}^{\infty}(0, \infty)$. Let $\bar{P}_{z}[S]=P_{z}[S]\left(x \partial_{x}-z\right)^{S(z)}$.

We assume in the sequel that all asymptotic characters are of infinite degree to simplify the statements.

Proposition 1. Suppose $m(S)=\infty . f \in \Gamma^{S}(0, \infty)$ iff $f \in C^{\infty}(0, \infty)$ and $P_{z}[S] f(t)=O\left(t^{z-\varepsilon}\right)$ for all $z \in \mathbb{C}$ and $\varepsilon>0$.

Proof. The functions $x^{k}(\ln x)^{j}$, for $\Re k \leq \Re z, 0 \leq j<S(k)$, form a complete set of solutions of the homogeneous ordinary differential equation $\bar{P}_{z}[S] f(t)=0$. A particular solution of $\bar{P}_{z}[S] f(t)=g(t)$, when $g(t)=O\left(t^{z+\varepsilon}\right)$, is $f_{0}(t)=\bar{Q}_{z}[S] g(t)$, where $\bar{Q}_{k}[S]=\prod_{\Re k^{\prime} \leq \Re k}, J_{k^{\prime}}^{S\left(k^{\prime}\right)}$ and $J_{k^{\prime}} \varphi(x)=x^{k^{\prime}} \int_{0}^{x} t^{-k^{\prime}-1} \varphi(t) d t$ (for $\varphi$ for which this integral exists); it is immediate that $\bar{Q}_{k}[S] \varphi(x)=O\left(x^{k+\delta}\right)$ if $\varphi(x)=O\left(x^{k+\delta}\right)$ for $\delta>0$ as $x \rightarrow 0$. The Proposition follows in both directions from these statements. 
Note that the space $C^{\infty}[0, \infty)$ is just $\Gamma^{S}(0, \infty)$ with $S(z)=1$ if $z \in \mathbb{Z}_{+}$ and $S(z)=0$ otherwise, and $P_{n}[S]=x^{n} \partial_{x}^{n}$ for any non-negative integer $n$.

Proposition 1 leads to a natural definition of $\Gamma^{\infty}$-spaces in several variables. Clearly, a proper setting for such spaces is a manifold with corners. A model for such a manifold is $\Omega=\left(\mathbb{R}_{+}\right)^{m} \times \mathbb{R}^{n-m}$. For this $\Omega$ let $\xi=\left(0, \ldots, 0, \xi_{m+1}, \ldots, \xi_{n}\right) \in \partial \Omega$. We say that $f\left(x_{1}, \ldots, x_{n}\right)$ is in $\Gamma^{\left(S_{1}, \ldots, S_{m} ; \infty\right)}(\Omega \mid \xi)$, for given asymptotic characters $S_{j}$ with $m\left(S_{j}\right)=\infty$, if there exists a neighborhood $N$ of $\xi$ in $\mathbb{R}^{n}$ such that

$$
\left(\prod_{i=1}^{m}\left(x_{i} \partial_{x_{i}}\right)^{s_{i}} P_{z_{i}}^{x_{i}}\left[S_{i}\right]\right)\left(\prod_{i=m+1}^{n} \partial_{x_{i}}^{s_{i}}\right) f(x)=O\left(\prod_{i=1}^{m} x_{i}^{z_{i}-\epsilon}\right)
$$

for all $x=\left(x_{1}, \ldots, x_{n}\right) \in \Omega \cap N$, for all $s_{i} \geq 0$, all $z_{i} \in \mathbb{C}$ and all $\epsilon>0$. For other points $\xi=\left(\xi_{1}, \ldots, \xi_{n}\right)$ of the boundary, we apply the same definition to $\Omega_{\xi}=\left(\mathbb{R}_{+}\right)^{m^{\prime}} \times \mathbb{R}^{n-m^{\prime}}$, where $m^{\prime}$ is the number of components among the $\xi_{1}, \ldots, \xi_{m}$ which are equal to zero and the coordinates are permuted so that these components are labeled $1, \ldots, m^{\prime}$. Globally, we say $f \in \Gamma^{\left(S_{1}, \ldots, S_{m} ; \infty\right)}(\Omega)$ if $f \in \Gamma^{\left(S_{i_{1}}, \ldots, S_{i_{m^{\prime}}} ; \infty\right)}(\Omega \mid \xi)$ for each $\xi \in \partial \Omega$, where $i_{1}, \ldots, i_{m^{\prime}}$ are the subscripts of exactly those components of the $\xi_{1}, \ldots, \xi_{m}$ which are equal to 0 . (The last entry ' $\infty$ ' in the superscript of $\Gamma$ denotes smoothness in the variables $\left.x_{m+1}, \ldots, x_{n}.\right)$ This definition is not the most natural but it is the simplest to state and it is equivalent to a definition that generalizes the notion of 'smoothness up to the boundary' [C3], in the sense of the existence of continuous extensions of derivatives from $\Omega$ to $\bar{\Omega}$; the latter employs a notion of local derivative operator $\partial^{k, j}[S]$ such that $\left(\partial^{k, j}[S] f\right)(0+)$ is the coefficient of $x^{k}(\ln x)^{j}$ for $f \in \Gamma^{\infty}\left(\mathbb{R}_{+}\right)^{1}$. We also note in passing that it is not coordinate invariant, although it does lead to a coordinate-invariant notion of $\Gamma^{\infty}$, which can then be defined on any manifold that looks like $\Omega$ near each boundary point [C3].

\footnotetext{
${ }^{1}$ Explicitly, let

$$
\tilde{\partial}^{k, \ell}[S]=\left(x^{k}(\ln x)^{-\ell}\right)^{-1} D_{k, \ell-1} \ldots D_{k,-S(k)+1} P_{k}[S],
$$

where $D_{k}=x \partial_{x}-k, D_{k, j}=\ln x D_{k}-j, P_{k}[S]=\prod_{\substack{\Re k^{\prime} \leq \Re k \\ k^{\prime} \neq k}} D_{k^{\prime}}^{S\left(k^{\prime}\right)}$. The $\partial^{k, j}[S]$ are just linear combinations of the $\tilde{\partial}^{k, \ell}[S]$ with coefficients depending on $S$. These are the anomalous derivative operators. The natural definition of anomalous smoothness is then: $f \in \Gamma^{\left(S_{1}, \ldots, S_{m} ; \infty\right)}(\Omega \mid 0)$ if there exists a neighborhood $N$ of 0 such that

$$
\left(\prod_{i=1}^{m} \partial_{x_{i}}^{z_{i}, j_{i}}\left[S_{i}\right]\right)\left(\prod_{i=m+1}^{n} \partial_{x_{i}}^{s_{i}}\right) f(x)
$$

extends to a continuous function on $\bar{\Omega} \cap N$, for all $z_{i} \in \mathbb{C}$ and $j_{i}, s_{i} \in \mathbb{Z}+$.
} 
As a simple application of these elementary ideas we have:

Proposition 2. Suppose $m\left(S_{j}\right)=\infty, f(x, y) \in \Gamma^{S_{1}, S_{2}}\left(\mathbb{R}_{+} \times \mathbb{R}_{+}\right)$, of bounded support in the first argument, and

$$
\left|P_{k}^{x}\left(S_{1}\right) f(x, y)\right| \leq(x y)^{\Re k-\varepsilon} h_{k, \varepsilon}(y)
$$

for all $k \in \mathbb{C}, \varepsilon>0$ and some $h_{k, \varepsilon}$ with $\int_{o}^{1} h_{k, \varepsilon}\left(\frac{1}{t}\right) \frac{d t}{t}<\infty$. Let $F(t)=$ $\int_{o}^{\infty} f\left(x, \frac{t}{x}\right) \frac{d x}{x}$. Then $F \in \Gamma^{S_{1}+S_{2}}\left(\mathbb{R}_{+}\right)$.

Proof. Assume first that $f(x, y)$ is of bounded support. Now $P_{z}\left[S_{1}+\right.$ $\left.S_{2}\right] F=P_{z}\left[S_{1}\right] P_{z}\left[S_{2}\right] F=\int_{o}^{\infty} P_{z}^{x}\left[S_{1}\right] P_{z}^{y}\left[S_{2}\right](f(x, y)) \frac{d x}{x}$, where $y=\frac{t}{x}$, since $F$ is also given by $\int_{o}^{\infty} f\left(\frac{t}{x}, x\right) \frac{d x}{x}$. Because $f$ is of bounded support, we find $P_{z}\left[S_{1}+S_{2}\right] F=\int_{0}^{B} O\left(x^{z-\frac{\varepsilon}{2}}\left(\frac{t}{x}\right)^{z-\varepsilon}\right) \frac{d x}{x}=O\left(t^{z-\varepsilon}\right)$ for all $z \in \mathbb{C}$ and $\varepsilon>0$. The result follows from Proposition 1.

In the complementary case where $f(x, y)$ is zero for $y$ near 0 , we have

$$
\begin{aligned}
\left|P_{z}\left[S_{1}\right] F(t)\right| & =\left|\int_{o}^{\infty} P_{z}^{x}\left[S_{1}\right](f(x, y)) \frac{d x}{x}\right| \\
& \leq \int_{0}^{B}\left|P_{z}^{x}\left(S_{1}\right) f(x, y) \frac{d x}{x}\right| \\
& \leq \int_{0}^{B}(x y)^{\Re z-\varepsilon} h_{z, \varepsilon}(y) \frac{d x}{x} \\
& =t^{\Re z-\varepsilon} \int_{0}^{B / t} h_{z, \varepsilon}(1 / y) \frac{d y}{y} \\
& \leq C \cdot t^{\Re z-\varepsilon}(1+\ln t),
\end{aligned}
$$

since $h_{z, \varepsilon}(y)$ can be chosen bounded for $y \leq 1$. $S_{1} \leq S_{1}+S_{2}, S_{1}+S_{2}$ is also an asymptotic character for $F$.

This proof completely bypasses the question of the evaluation of the asymptotic coefficients of $F(t)$ as $t \rightarrow 0+$. An equally simple argument can be given using Mellin transforms, but in the present context the hypotheses on $f$ are much more transparent. Formulas can be written down using either the present calculus [C3] or Mellin transforms, but this is a more complicated affair. Other examples of applications are given in a lengthier article.

\section{Examples of applications of singular heat expansions}

Even though the overall expansion (1) is far more complex than in the smooth case, it turns out that, somewhat surprisingly, some crucial terms in the $w_{k j}(\varphi)$ are far easier to compute to all orders than the 'classical' $u_{k}(\varphi)$. This was done in [C5] for a Schrödinger operator on the circle, with 
one isolated singularity at $x=0$ with respect to a local coordinate, near which the potential is of the form $\psi(|x|) /|x|+\psi_{1}(x)$, with $\psi \in C^{\infty}[0, \infty)$, $\psi_{1} \in C^{\infty}(\mathbb{R})$ and $\psi_{1}^{(k)}(0)=0$ for all $k$. The spectral information so obtained is sufficient to prove that $\psi^{(k)}(0)$ are determined for all $k$ by the spectrum of the Dirichlet operator if $\psi(0) \neq 0$.

In [C5] it is conjectured that similar results hold for operators with one irregular singular point. The proof would require calculations of asymptotic coefficients for the expansion that we discuss in Section 3.

As a simple example of an index problem where it is enough to know just that (1) exists, consider $L=i \partial_{x}+i \psi(x)$ on the interval $(0,1)$, with $\psi(x)$ a hermitian matrix of smooth $n \times n$ functions, such that $\psi(x)=A_{y}(x) /$ $|x-y|^{\alpha_{y}}$ for $x$ near $y$ when $y$ is 0 or 1 , for some $\alpha_{y}>1$, and matrices $A_{y}(x)$ such that $B_{y}=\lim _{x \rightarrow y} A(x)$ exists and is a nonsingular matrix. Suppose that we know that (1) exists for operators of the form $H=-\partial_{x}^{2}+v(x)$ on $(0,1)$ with $v(x)$ in $\Gamma^{\infty}$ at 0 and 1 , the singularities at 0 and 1 being irregular; this is true, according to the next section. $\left(L^{*} L\right.$ and $L L^{*}$ are of the form of such $H$.) Suppose further that these heat expansions are local, in the sense that

$$
\begin{aligned}
& \text { If } U \text { is an open set and } H_{1}=H_{2} \text { on } C^{\infty}(U) \text {, while } \varphi \in L^{\infty} \\
& \text { and supp } \varphi \subset \bar{U} \text {, we have } \operatorname{Tr} \varphi\left(e^{-t H_{1}}-e^{-t H_{2}}\right)=O\left(t^{k}\right) \text { for } \\
& \text { all } k \text {, }
\end{aligned}
$$

which is also true, as might be expected by what is well-known for classical expansions [Gi]. Then one can prove by a short argument that the index of $L$, which can be defined as a closed operator, is given by $\frac{1}{2}\left(\operatorname{sgn} B_{0}-\operatorname{sgn} B_{1}\right)$, where 'sgn' denotes the signature of a matrix, i.e., the number of negative minus the number of positive eigenvalues. To sketch the argument, use the notation $\left(\mathcal{D}_{k, j} f\right)(a)$ for the coefficient of $|x-a|^{k}(\ln |x-a|)^{j}$ in the asymptotic expansion as $x \rightarrow a$ for a function $f$ which is in $\Gamma^{\infty}$ at $a$, the latter being an endpoint of the interval of definition of $f$. For an operator $H$ of the kind described above, the local nature of the heat expansion implies that

$$
\begin{aligned}
\left.\mathcal{D}_{0,0}^{t} \operatorname{Tr} e^{-t H}\right|_{t=0}= & F_{0}\left(\left\{\mathcal{D}_{k, j} v(0)\right\}_{k \in \mathbb{C}, j \in \mathbb{Z}_{+}}\right) \\
& +F_{1}\left(\left\{\mathcal{D}_{k, j} v(1)\right\}_{k \in \mathbb{C}, j \in \mathbb{Z}_{+}}\right) .
\end{aligned}
$$

Hence, by the well-known formula for the index in terms of heat invariants,

$$
\begin{aligned}
& \text { index } L=\operatorname{Tr}\left(e^{-t L^{*} L}-e^{-t L L^{*}}\right)=\Phi_{0}\left(\left\{\mathcal{D}_{k, j} A_{0}(0)\right\}_{k \in \mathbb{C}, j \in \mathbb{Z}_{+}}\right) \\
& +\Phi_{1}\left(\left\{\mathcal{D}_{k, j} A_{1}(1)\right\}_{k \in \mathbb{C}, j \in \mathbb{Z}_{+}}\right) \text {. }
\end{aligned}
$$


By the invariance of the index under small perturbations it follows that, in fact, $\Phi_{y}=\Phi_{y}\left(\left(\mathcal{D}_{0,0} A_{y}\right)(y)\right)$, if we deform $L$ to constant $A_{y}(x)$ in sufficiently small neighborhoods of the points $y$ (see Proposition A.1 for the required estimate). Apply the index formula above to the two operators $L_{j}=i \partial_{x}+i \psi_{j}(x)$, where

$$
\begin{aligned}
& \psi_{0}(x)=\frac{B_{0}}{|x|^{\alpha_{0}}} \chi_{0}(x)+\frac{1_{n}}{|1-x|^{\alpha_{1}}} \chi_{1}(x), \\
& \psi_{1}(x)=\frac{1_{n}}{|x|^{\alpha_{0}}} \chi_{0}(x)+\frac{B_{1}}{|1-x|^{\alpha_{1}}} \chi_{1}(x)
\end{aligned}
$$

(where $\chi_{0} \in C_{0}^{\infty}\left[0, \frac{1}{2}\right)$, with $\chi_{0} \equiv 1$ near 0 , and $\chi_{1} \in C_{0}^{\infty}\left(\frac{1}{2}, 1\right]$, with $\chi_{1} \equiv 1$ near 1 ) and compute the index directly by solution of decoupled first order linear differential equations to obtain

$$
\begin{aligned}
& \frac{1}{2}\left(n+\operatorname{sgn} B_{0}\right)=\operatorname{index} L_{0}=\Phi_{0}\left(B_{0}\right)+\Phi_{1}\left(1_{n}\right) \\
& -\frac{1}{2}\left(n+\operatorname{sgn} B_{1}\right)=\operatorname{index} L_{1}=\Phi_{1}\left(B_{1}\right)+\Phi_{0}\left(1_{n}\right)
\end{aligned}
$$

Adding, we obtain index $L=\frac{1}{2}\left(\operatorname{sgn} B_{0}-\operatorname{sgn} B_{1}\right)$, if we use the fact that $\Phi_{0}\left(1_{n}\right)+\Phi_{1}\left(1_{n}\right)=0$, again by a direct index calculation.

\section{Heat asymptotics for an operator with an irregular singular point on $\mathbb{R}$}

The machinery outlined in Section 1 can be used to tackle the question of the existence of (1) for semibounded uniformly elliptic differential operators of even order with isolated irregular singularities, when each coefficient is of the form $b\left(|x-y|, \frac{(x-y)}{|x-y|}\right)$ for $x$ in the neighborhood of the typical singular point $y$, with $b(r, \theta)$ a function in $\Gamma^{\infty}\left(\mathbb{R}_{+} \times S^{n-1}\right)$.

As an example we will give the salient points of the proof of the existence of a power-log asymptotic expansion for a model operator with an irregular singularity, $H=-\partial_{x}^{2}+x^{-\alpha}(\kappa+v(x))$ on $\mathbb{R}_{+}$where $\alpha>2, \kappa>0, v(x)$ is a real-valued function in $\Gamma_{o}^{\infty}\left(\mathbb{R}_{+}\right)$and $v(0)=0$. Under these assumptions, $H$ is essentially self-adjoint on $C_{o}^{\infty}(0, \infty)[\mathrm{RS}]$, so that it extends uniquely to an unbounded self-adjoint operator on $L^{2}\left(\mathbb{R}_{+}\right)$, also denoted by $H$. The heat semigroup $e^{-t H}$ can be defined by the spectral theorem and it is easy to show that it is represented by a kernel function, $K_{t}(x, y)$, which is smooth in $(t, x, y) \in\left(\mathbb{R}_{+}\right)^{3}$, by solving a heat equation. Using the inequality $H^{2} \geq \partial_{x}^{4}+\frac{c}{x^{2 \alpha}}-B$ on $C_{0}^{\infty}(0, \infty)$ for some constants $c>0$ and $B \in \mathbb{R}$, we can show that, for each $\varepsilon>0$,

$$
\left\|x^{-\beta} \partial_{x}^{d} R_{\lambda}\right\|=O\left(|\lambda|^{-1+\frac{\beta}{\alpha}+\frac{d}{2}}\right) \text { as }|\lambda| \rightarrow \infty \text { for } \Re \lambda<\varepsilon|\Im \lambda|
$$


where $R_{\lambda}=(\lambda-H)^{-1},\|\cdot\|$ denotes operator norm on $L^{2}(0, \infty)$ and $\frac{\beta}{\alpha}+$ $\frac{d}{2}<1$. Further, using a comparison of the resolvents of $H$ and $-\partial_{x}^{2}$ (which is possible because of the essential self-adjointness of $H$ on $\left.C_{o}^{\infty}(0, \infty)\right)$, we can show that

$$
\left\|\phi R_{\lambda}\right\|_{H S} \leq C \cdot|\lambda|^{-\frac{3}{4}}\|\phi\|_{L^{2}}
$$

for $\lambda$ as in (1), with $\|\cdot\|_{H S}$ denoting Hilbert-Schmidt norm and $\phi \in$ $L^{2}(0, \infty)$. (Details of the proofs of these estimates are given in the appendix.)

The following theorem is the main application of the machinery of Section 1 that we include in this note.

Theorem 1. There exists an asymptotic character $S$ of infinite degree with $S(z)=0$ for $\Re z \leq \frac{1}{2}$ such that, for each $\varphi \in C_{o}^{\infty}[0, \infty)$,

$$
\int_{o}^{\infty} \varphi(x) K_{t}(x, x) d x \sim \frac{1}{(4 \pi t)^{\frac{1}{2}}} \int_{o}^{\infty} \varphi(x) d x+\sum_{z \in \mathbb{C}} \sum_{0 \leq j<S(z)} u_{z j}(\varphi) t^{z}(\ln t)^{j}
$$

for some $u_{z j} \in \mathcal{D}^{\prime}(\mathbb{R})$ with supp $u_{z j} \subset[0, \infty)$.

The asymptotic character $S$ depends of course on the potential $v$. In special cases $S$ can be simply determined as a corollary of the following type of theorem.

Theorem 2. Let $f(\xi, \eta) \equiv f_{H}(\xi, \eta)$ be defined for $\xi>0, \eta>0$ by $f\left(\frac{t^{\frac{1}{\alpha}}}{x}, x\right)=t^{\frac{1}{2}} e^{-t H}(x, x)$. For $H_{\kappa, \alpha}=-\partial_{x}^{2}+\frac{\kappa}{x^{\alpha}}$ on $L^{2}\left(\mathbb{R}_{+}\right), \kappa>0$, $\alpha>2, f \in \Gamma^{S_{1}, S_{2}}\left(\mathbb{R}_{+} \times \mathbb{R}_{+}\right)$, where $S_{1}(j \alpha)=1=S_{2}\left(j\left(\frac{\alpha}{2}-1\right)\right)$ for $j=0,1,2, \ldots$, while $S_{1}(z)=S_{2}(z)=0$ for other values of $z$. Furthermore, $\left|P_{z}^{\eta}\left[S_{1}\right] f(\xi, \eta)\right| \leq C \cdot(\xi \eta)^{\Re z}$ for each $z \in \mathbb{C}$.

Theorem 1, for $H=H_{\kappa, \alpha}$, is then an easy corollary of Theorem 2, if Proposition 2 is used. $S$ in this case is $S_{1}+\widetilde{S}_{2}$ where

$$
\widetilde{S}_{2}(z)=\max _{j \in \mathbb{Z}_{+}} S_{2}(z-j) .
$$

For general $H$, Theorem 1 is again a corollary of a result like Theorem 2 , but we will limit ourselves to $H_{\kappa, \alpha}$ here, because the computations are quite a bit longer in the general case. The procedure would be identical for arbitrary $H$-we would just have more terms to keep track of. 
Sketch of the proof of Theorem 2. Let $\xi=\frac{t^{\frac{1}{\alpha}}}{x}, \eta=x$. Then $\eta \partial_{\eta}=\alpha t \partial_{t}+$ $x \partial_{x}, \xi \partial_{\xi}=t \partial_{t}$. We compute $P_{z}\left[S_{2}\right] f$. For $z=\frac{\alpha}{2}-1, P_{z}\left[S_{2}\right]=\eta \partial_{\eta}$. Use the fact that, if $K$ is an integral operator with smooth kernel $K(x, y)$, $\partial K(x, x) / \partial x=[\partial, K](x, x)$ and $\left[x \partial_{x}, K\right](x, x)=K(x, x)+x\left[\partial_{x}, K\right](x, x)$. Applying $t \partial_{t}$ directly under the integral sign in the well-known formula $e^{-t H}=\int_{C} \frac{d \lambda}{2 \pi i} e^{-t \lambda} R_{\lambda}$ (where $C$ is a cone shaped contour enclosing the positive real axis), we find

$$
\eta \partial_{\eta} f(\xi, \eta)=t^{\frac{1}{2}} \int \frac{d \lambda}{2 \pi i}\left(\frac{\alpha}{2} R_{\lambda}+\alpha R_{\lambda} H R_{\lambda}+x\left[\partial_{x}, R_{\lambda}\right]\right)(\eta, \eta) .
$$

It is in no way clear that this is $O\left(\eta^{\frac{\alpha}{2}-1}\right)$. The trick that makes the calculation work is writing

$$
H=-\frac{1}{2}\left[x \partial_{x}, H\right]+\frac{1}{2}(2-\alpha) \kappa / x^{\alpha} .
$$

Using the fact that $\left[\partial_{x}, R_{\lambda}\right]=-R_{\lambda} \frac{\alpha \kappa}{x^{\alpha+1}} R_{\lambda}$ (note that the factors can be grouped into subproducts that form bounded operators), we find

$\eta \partial_{\eta} f(\xi, \eta)=t^{\frac{1}{2}} \int \frac{d \lambda}{2 \pi i} e^{-t \lambda}\left[-\left(1-\frac{\alpha}{2}\right) x R_{\lambda} \frac{\alpha \kappa}{x^{\alpha+1}} R_{\lambda}+R_{\lambda} \frac{\alpha \kappa\left(1-\frac{\alpha}{2}\right)}{x^{\alpha}} R_{\lambda}\right](\eta, \eta)$.

Finally using $x R_{\lambda}=R_{\lambda} x+2 R_{\lambda} \partial_{x} R_{\lambda}$, we arrive at ${ }^{2}$

$$
\eta \partial_{\eta} f(\xi, \eta)=\alpha \kappa(\alpha-2) t^{\frac{1}{2}} \int \frac{d \lambda}{2 \pi i} e^{-t \lambda}\left(R_{\lambda} \partial_{x} R_{\lambda} \frac{1}{x^{\alpha+1}} R_{\lambda}\right)(\eta, \eta) .
$$

This formula will give the required estimates; we integrate against a $\varphi(\eta) \in$ $L^{1}$, write $\varphi=\varphi_{1} \varphi_{2}$, with $\varphi_{j} \in L^{2}$, and apply (2) and (3) to get $\left|P_{z}^{\eta}\left[S_{2}\right] f(\xi, \eta)\right| \leq$ $C \cdot|\xi \eta|^{z}$, for $z=\frac{\alpha}{2}-1 .^{3}$ We proceed to apply $\eta \partial_{\eta}-j\left(\frac{1}{2}-\frac{1}{\alpha}\right)$ successively for $j=1,2, \ldots$ to obtain the same result for arbitrary $z$. There are technical problems, but it all works out within the same scheme. An outline is given below.

\footnotetext{
${ }^{2}$ The value of the computations that lead to (5) lies, in part, in the fact that they can be localized in $x$; if we only assume, for example, that $v(x) \equiv 0$ for $x \leq a$, without even smoothness assumptions for $x$ away from 0 , we can use $\chi(x) x \partial_{x}$ in (4) instead of $x \partial_{x}$ (with suitable modifications in the other terms), with $\chi \in C_{0}^{\infty}[0, a / 2]$, and $\chi(x) \equiv 1$ for $x$ near 0 , and obtain, instead of (5),

$\eta \partial_{\eta} f(\xi, \eta)=\alpha \kappa(\alpha-2) t^{\frac{1}{2}} \int \frac{d \lambda}{2 \pi i} e^{-t \lambda}\left(R_{\lambda} \chi(x) \partial_{x} R_{\lambda} \chi(x) \frac{1}{x^{\alpha+1}} R_{\lambda}\right)(\eta, \eta)+O\left(t^{k}\right), \quad \forall k$,

when $\eta \leq a / 2$. The same can be done in higher dimension and systematically to all orders.

${ }^{3}$ See also [E2], where this idea is applied to obtain uniform estimates, with respect to parameters, for singular Green's functions that arise in the linearization of the equations for the harmonic map between asymptotically hyperbolic manifolds.
} 
The estimates for the $\xi$-derivatives of each of $P_{z}^{\eta}\left[S_{2}\right] f(\xi, \eta)$ can be reduced to classical heat expansions for operators with smooth coefficients. One way to make this explicit is to use a local version of (4), with $x \partial_{x}$ on the right-hand side replaced by $\chi(x) x \partial_{x}$, when $x=0$ is now thought of as the typical point where the coefficients of $H$ are smooth, and with $\chi$ a $C_{0}^{\infty}$ function which is identically 1 near $x=0$ and has support that does not extend as far as the nearest singularity. This approach gives sharp estimates on the $\xi$-derivatives.

The higher $\eta$-'derivatives'. In the formula for the general $P_{z}\left[S_{2}\right]$ we will encounter operators of the form

$$
\Pi=\Pi_{k}\left(\alpha_{1}, \ldots, \alpha_{k} ; \lambda\right)=x^{-\alpha_{1}} \partial_{x}^{d_{1}} R_{\lambda} \ldots x^{-\alpha_{k}} \partial_{x}^{d_{k}} R_{\lambda}
$$

where each $d_{j}$ is 0 or 1 and $\Re \alpha_{j} \leq 0$. We call a formal expression of the form (6) a resolvent product. We will also use this term for the operator given by (6) if the actual product in the sense of operators is defined as a bounded operator on $L^{2}$. It is clear that $\Pi$ is a bounded operator if, for each $j, \frac{\alpha_{j}}{\alpha}+\frac{d_{j}}{2} \leq 1$. Less obvious is the following fundamental result:

Proposition 3. The operator (6) is a bounded-operator-valued holomorphic function of $\alpha_{1}, \ldots, \alpha_{k}$ for $\Re \alpha_{j}>0,1 \leq j \leq k$, and $\Re i(\Pi)>0$, where $i(\Pi)$ is the decay index of $\Pi$, defined by $i(\Pi)=k-\sum_{j=1}^{k}\left(\frac{d_{j}}{2}+\frac{\alpha_{j}}{\alpha}\right)$. For $\lambda$ sufficiently large in $\Re \lambda<\varepsilon|\Im \lambda|$ (for any given $\varepsilon>0$ ), the operator norm of $\Pi(\lambda)$ is $O\left(|\lambda|^{-\Re i(\Pi)}\right)$.

The proof is not difficult; the exponents $\alpha_{j}$ are redistributed by commuting factors of the form $x^{-r}$ through the $R_{\lambda}$ 's. The operator norm estimate follows from (2). (A more formal treatment of resolvent products and details of the proof of Proposition 3 are given in the appendix.)

Lemma 1. Let $\Pi=\Pi(\lambda)$ be a resolvent product with $i(\Pi)>0$. Then

$$
\left[\alpha t \partial_{t}+x \partial_{x}-\alpha(i(\Pi)-1)\right] t^{\frac{1}{2}} \int_{C} \frac{d \lambda}{2 \pi i} e^{-t \lambda} \Pi(\lambda)(\eta, \eta)
$$

is a linear combination of terms of the form $\int_{C} \frac{d \lambda}{2 \pi i} e^{-t \lambda} \tilde{\Pi}(\lambda)(\eta, \eta)$ where $i(\tilde{\Pi}(\lambda))=i(\Pi)+\frac{1}{2}-\frac{1}{\alpha}$.

This is proved by using the ideas that led to the derivation of the formula (5) for $\eta \partial_{\eta} f(\xi, \eta)$. 
From this lemma it follows that $\left(\prod_{j=0}^{k-1}\left[\eta \partial_{\eta}-j\left(\frac{1}{2}-\frac{1}{\alpha}\right)\right]\right) f(\xi, \eta)$ is a linear combination of terms of the form $t^{\frac{1}{2}} \int \frac{d \lambda}{2 \pi i} e^{-t \lambda} \Pi(\lambda)(\eta, \eta)$ with $i(\Pi(\lambda))=$ $k\left(\frac{1}{2}-\frac{1}{\alpha}\right)+1$. Now we can use (2) and (3) to show that if $\varphi \in L^{1}$,

$$
\mid \int \varphi(x)\left(\left.R_{\lambda} \Pi(\lambda) R_{\lambda}(x, x) d x\left|\leq C \cdot\|\varphi\|_{L^{1}} \cdot\right| \lambda\right|^{-\frac{3}{2}-i(\Pi)}\right.
$$

for $|\lambda| \rightarrow \infty, \Re \lambda \geq \varepsilon \cdot|\Im \lambda|$. It follows easily that, for $z=\alpha k \cdot\left(\frac{1}{2}-\frac{1}{\alpha}\right)$,

$$
\int_{o}^{\infty} P_{z}^{\eta}\left[S_{2}\right] f(\xi, \eta) \varphi(\eta) d \eta \leq C \cdot\|\varphi\|_{L^{1}} \cdot t^{i(\Pi)-1}
$$

so that $\left|P_{z}^{\eta}\left[S_{2}\right] f(\xi, \eta)\right| \leq C \cdot|\xi \eta|^{\alpha k \cdot\left(\frac{1}{2}-\frac{1}{\alpha}\right)}=C \cdot|\xi \eta|^{z}$.

\section{Extensions and discussion}

The example that was discussed in detail in Section 3 is not just a model for an irregular singularity, it is also the building block for the analysis of operators of the form $-\partial_{x}^{2}+A / x^{\alpha}$, where $A$ is a differential operator. Such operators arise as Laplacians on manifolds with cusps. Following the ideas of [C2], this problem is reduced to the study of the behavior of $\operatorname{Tr} \varphi e^{-t H_{\kappa}}$ as a function of $t$ and $\kappa$ [C4] for $H_{\kappa}=-\partial_{x}^{2}+\frac{\kappa}{x^{\alpha}}(\alpha \geq 2)$. This question in turn can be tackled by employing the calculus of Section 1 .

Besides operators with irregular singularities at finite points, the method outlined in this proof also covers operators on $\mathbb{R}^{n}$ with an irregular singularity at $\infty$, which would include, in particular, operators of the form $-\Delta_{n}+v(x) \cdot|x|^{\alpha}$, (with $v(x) \geq c>0$ for $|x|$ near $\infty$ ) where $v(x)$ is bounded, with sufficient smoothness conditions at $\infty$. Details will appear elsewhere.

The calculus of Section 1 is developed in more detail in [C3]. The calculation of the asymptotic coefficients of $\operatorname{Tr} \varphi e^{-t H}$ — which entails evaluating (5) at $\eta=0$, for instance - requires a whole new bag of tricks. This is done in the longer version of this paper, although the main ideas are in [C4].

We conjecture that operators of the form $-\Delta_{n}+v(x) / p(x)$, where $v(x) \in$ $C_{0}^{\infty}\left(\mathbb{R}^{n}\right)$ and $p(x)$ is a polynomial, have heat asymptotics similar to the operators discussed here. Some progress has been made for special $p(x)$.

\section{Acknowledgements}

This work has been supported by NSF and the Case RIG. The support of the Research Center of Crete in Iraklion, Crete, where the work was completed in the Summer of 1989, is also gratefully acknowledged. Conversations with Carlos Corona have been helpful. 
A preprint of this work was produced and distributed in November of 1989. Since then Melrose has published an article on a "calculus of conormal distributions" [Me] which is closely related to the ideas of Section 1. See also [E2, Ma]. The $\Gamma^{\infty}$ spaces of this paper are just the "polyhomogeneous functions" [Me, Ma, E2]. The "singular asymptotics lemma" is closely related to the "push-forward formula." I am grateful to Michael Economakis and Rafe Mazzeo for discussions on the relationship of this paper to Melrose's and their work, as well as for critically reading the manuscript during my stay at the University of Washington during the Winter and Spring Quarters of 1993.

\section{Appendix A. Proofs of the technical lemmas}

Resolvent Estimates. In this Section we will give proofs of some of the operator norm and trace ideal estimates on the resolvents of the operators studied in this paper. The fundamental estimate is

Lemma A.1. Let $\kappa>0$ and $\alpha>2$ be fixed and let $H_{\kappa, \alpha}$ be the operator

$$
C_{0}^{\infty}(0, \infty) \ni \varphi \mapsto H_{\kappa, \alpha} \varphi=-\varphi^{\prime \prime}+\kappa x^{-\alpha} \varphi \in C_{0}^{\infty}(0, \infty)
$$

There exists constants $c>0$ and $B \in \mathbb{R}$ such that

$$
\left\|H_{\kappa, \alpha} \varphi\right\|^{2} \geq\left\|\partial_{x}^{2} \varphi\right\|^{2}+c \cdot\left\|x^{-\alpha} \varphi\right\|^{2}-B\|\varphi\|^{2}
$$

for all $\varphi \in C_{0}^{\infty}(0, \infty)$.

Proof. We start with the operator identity

$$
\left(H_{\kappa, \alpha}\right)^{2}=\partial_{x}^{4}-2 \kappa \partial_{x} x^{-\alpha} \partial_{x}-\frac{\kappa \alpha(\alpha+1)}{x^{\alpha+2}}+\frac{\kappa^{2}}{x^{2 \alpha}} .
$$

Proceeding a little further, we write

$$
-\partial_{x} x^{-\alpha} \partial_{x}=x^{-\alpha / 2}\left(-\partial_{x}^{2}\right) x^{\alpha / 2}+\frac{\alpha}{2}\left(\frac{\alpha}{2}+1\right) x^{-\alpha-2} .
$$

Now use the classical Hardy inequality $\left\|\partial_{x} \varphi\right\|^{2} \geq \frac{1}{4}\left\|x^{-1} \varphi\right\|^{2}$ for $\varphi \in$ $C_{0}^{\infty}(0, \infty)[\mathrm{C} 2]$ to obtain

$$
\left\|H_{\kappa, \alpha} \varphi\right\|^{2} \geq\left\|\partial_{x}^{2} \varphi\right\|^{2}+\frac{\kappa}{2}\left(1-\alpha^{2}\right)\left\|x^{-\frac{\alpha}{2}-1} \varphi\right\|^{2}+\kappa^{2}\left\|x^{-\alpha} \varphi\right\|^{2} .
$$

Since $x^{-\alpha-2} \leq \varepsilon \cdot x^{-2 \alpha}+B_{\varepsilon}$, for $\alpha>2$, the estimate of the lemma follows.

From this a priori estimate follows: 
Proposition A.1. Let $\bar{H}_{\kappa, \alpha}$ denote the unique self-adjoint extension of the operator $H_{\kappa, \alpha}$ of Lemma A.1, viewed as an unbounded operator on $L^{2}(0, \infty)$. For fixed $\kappa$ and $\alpha$, let $R_{\lambda}=\left(\lambda-\bar{H}_{\kappa, \alpha}\right)^{-1}$, the resolvent of $\bar{H}_{\kappa, \alpha}$ for $\lambda \in \mathbb{C} \backslash[0, \infty)$. Let $\beta \geq 0$ and $d=0$ or 1 or 2 , and suppose that $\frac{\beta}{\alpha}+\frac{d}{2} \leq 1$. Then the operator

$$
B_{\beta, d, \lambda}=x^{-\beta} \partial_{x}^{d} R_{\lambda},
$$

which is defined on the dense domain $\left(\lambda-\bar{H}_{\kappa, \alpha}\right) C_{0}^{\infty}(0, \infty)$, extends uniquely to a bounded operator on $L^{2}(0, \infty)$ and the operator norm satisfies

$$
\left\|B_{\beta, d, \lambda}\right\| \leq C_{\varepsilon} \cdot|\lambda|^{-1+\frac{\beta}{\alpha}+\frac{d}{2}} \text { for } \Re \lambda<\varepsilon|\operatorname{Im} \lambda|-\varepsilon .
$$

Proof. Let $D=\left(\lambda-\bar{H}_{\kappa, \alpha}\right) C_{0}^{\infty}(0, \infty)$ and $\varphi \in D$, so that $\varphi=\left(\lambda-\bar{H}_{\kappa, \alpha}\right) \psi$ for some $\psi \in C_{0}^{\infty}(0, \infty)$. Then $\left\|B_{\beta, d, \lambda} \varphi\right\|^{2}=\left(\psi, \partial_{x}^{d} x^{-2 \beta} \partial_{x}^{d} \psi\right)$. If $d=2$, we must have $\beta=0$, and

$$
\left\|B_{\beta, d, \lambda} \varphi\right\|^{2}=\left(\psi, \partial_{x}^{4} \psi\right) \leq\left\|H_{\kappa, \alpha} \psi\right\|^{2}+B \cdot\|\psi\|^{2},
$$

by Lemma A.1. The estimate follows in this case, since

$$
\left\|H_{\kappa, \alpha} \psi\right\|^{2}=\left\|\bar{H}_{\kappa, \alpha} R_{\lambda} \varphi\right\|^{2} \leq c_{\varepsilon}\|\varphi\|^{2} \text {, when } \Re \lambda<\varepsilon \cdot|\operatorname{Im} \lambda|-\varepsilon,
$$

by the spectral theorem. So assume now $d=0$ or 1 . Use the commutator

$$
\left[\partial_{x}^{d}, x^{-2 \beta}\right]=-d \cdot 2 \beta \cdot x^{-2 \beta-1},
$$

to write

$$
\begin{aligned}
\left\|B_{\beta, d, \lambda} \varphi\right\|^{2} & =\Re\left(\psi, \partial_{x}^{d} x^{-2 \beta} \partial_{x}^{d} \psi\right) \\
& =\Re\left(\alpha^{-2 \beta} \psi, \partial_{x}^{2 d} \psi\right)-2 d \cdot \beta \cdot \Re\left(x^{-2 \beta-1} \psi, \partial_{x}^{d} \psi\right)
\end{aligned}
$$

For $d=0$ use the inequality

$$
\left(\psi, x^{-2 \beta} \psi\right) \leq \delta \cdot \frac{\beta}{\alpha} \cdot\left\|x^{-\alpha} \psi\right\|^{2}+\delta^{-\frac{\beta}{\alpha-\beta}} \cdot \frac{\alpha-\beta}{\alpha} \cdot\|\psi\|^{2}
$$

(which follows from $a b \leq \frac{a^{p}}{p}+\frac{b^{q}}{q}$, with $p^{-1}+q^{-1}=1$, if we choose $p=$ $\left.\frac{\alpha}{\beta}, a=\delta^{1 / p} x^{-2 \beta}, b=\delta^{-1 / p}\right)$. We have already shown that $\left\|x^{-\alpha} \psi\right\|^{2} \leq$ $c_{\varepsilon} \cdot\|\varphi\|^{2}$ and, since $\|\psi\|^{2} \leq c_{\varepsilon} \cdot|\lambda|^{-2} \cdot\|\varphi\|^{2}$, if we choose $\delta=\delta^{-\frac{\beta}{\alpha-\beta}}|\lambda|^{-2}$, we obtain

$$
\left(\psi, x^{-2 \beta} \psi\right) \leq c_{\varepsilon} \cdot|\lambda|^{-2\left(1-\frac{\beta}{\alpha}\right)} .
$$

This is turn yields

$$
\left\|B_{\beta, d, \lambda} \varphi\right\| \leq C_{\varepsilon} \cdot|\lambda|^{-1+\frac{\beta}{\alpha}} \cdot\|\varphi\|,
$$


which proves the estimate in this case as well. The result also follows along similar lines for $\beta=0$, if we use

$$
\left(\psi, \partial_{x}^{2} \psi\right) \leq \frac{1}{2}\left(\delta \cdot\left\|\partial_{x}^{2} \psi\right\|+\delta^{-1}\|\psi\|^{2}\right)
$$

the estimate of the Proposition for $d=2$, and choose $\delta=|\lambda|^{-1 / 2}$.

For the remaining general case, with $d=1$ and $\beta \leq \frac{\alpha}{2}$, use

$$
\left\|B_{\beta, d, \lambda} \varphi\right\|^{2} \leq \frac{1}{2}\left(\delta \cdot\left\|B_{2 \beta, 0, \lambda} \varphi\right\|^{2}+\delta^{-1} \cdot\left\|B_{0,2 d, \lambda} \varphi\right\|^{2}\right)+\left\|B_{\beta+1,0, \lambda} \varphi\right\|^{2}
$$

which follows from A.1 for any $\delta>0$, the estimates just established for the cases $\beta=0$ and $d=0$, and choose $\delta=|\lambda|^{-2 \beta / \alpha+d}$.

The following Proposition provides a convenient tool in establishing some of the operator estimates needed for our problem. It gives a comparison of the resolvent of $H_{\kappa, \alpha}$ to the resolvent of the "free" Laplacian $H_{o}=-\partial_{x}^{2}$ on $\mathbb{R}$, for which the resolvent is an integral operator with kernel function

$$
\left(\lambda-H_{o}\right)^{-1}(x, y)=\frac{1}{2 \sqrt{-\lambda}} e^{-\sqrt{-\lambda}|x-y|} \quad(\Re \sqrt{-\lambda}>0)
$$

Because the operators of interest to us act on functions on $(0, \infty)$, we need the projection

$$
\Pi:\left.L^{2}(-\infty, \infty) \ni f \mapsto f\right|_{[0, \infty)} \in L^{2}(0, \infty) .
$$

Proposition A.2. Let $R_{\lambda}^{o}=\Pi \cdot\left(\lambda-H_{0}\right)^{-1} \Pi$. Let $Q_{\kappa, \alpha, \lambda}=\kappa \cdot x^{-\alpha}(\lambda-$ $\left.\bar{H}_{\kappa, \alpha}\right)^{-1}$, which is defined as a bounded operator by Proposition A.1. Then

$$
R_{\lambda}=\left(\lambda-\bar{H}_{\kappa, \alpha}\right)^{-1}=R_{\lambda}^{o}+R_{\lambda}^{o} Q_{\kappa, \alpha, \lambda}
$$

Proof. Let $D=\left(\lambda-\bar{H}_{\kappa, \alpha}\right) C_{0}^{\infty}(0, \infty)$, which is dense in $L^{2}(0, \infty)$. For $\varphi \in$ $D$ we have $\varphi=\left(\lambda-\bar{H}_{\kappa, \alpha}\right) \psi$ with $\psi \in C_{0}^{\infty}(0, \infty)$. Let $D_{0}=\left(\bar{\lambda}-H_{0}\right) C_{0}^{\infty}(\mathbb{R})$, which is dense in $L^{2}(\mathbb{R})$ because $-\partial_{x}^{2}$ is essentially self-adjoint on $C_{0}^{\infty}(\mathbb{R})$. Let $\varphi_{0}=\left(\bar{\lambda}-H_{0}\right) \psi_{0}$ be an arbitrary element of $D_{0}$. Then

$$
\left(\Pi \varphi_{0}, R_{\lambda} \varphi\right)=\left(\Pi\left(\bar{\lambda}-H_{0}\right) \psi_{0}, \psi\right) .
$$

Integrating by parts twice, we obtain

$$
\begin{array}{r}
\left(\Pi \varphi_{0}, R_{\lambda} \varphi\right)=\left(\Pi \psi_{0},\left(\lambda-\bar{H}_{\kappa, \alpha}\right) \psi\right) \\
+\left(\Pi \psi_{0}, \frac{\kappa}{x^{\alpha}} \psi\right) .
\end{array}
$$


Adding and subtracting $\frac{\kappa}{x^{\alpha}}$ causes no problems because $\psi \equiv 0$ near $x=0$. Now $\frac{\kappa}{x^{\alpha}} \psi=Q_{\kappa, \alpha, \lambda} \varphi$, by the definition of $Q_{\kappa, \alpha, \lambda}$, so that

$$
\begin{aligned}
\left(\Pi \varphi_{0}, R_{\lambda} \varphi\right)= & \left(\Pi\left(\bar{\lambda}-H_{0}\right)^{-1} \varphi_{0}, \varphi\right) \\
& +\left(\Pi \cdot\left(\bar{\lambda}-H_{0}\right)^{-1} \varphi_{0}, Q_{\kappa, \alpha, \lambda} \varphi\right) .
\end{aligned}
$$

The resolvent comparison formula follows.

The main application of this formula will be the Hilbert-Schmidt estimate of Proposition A.3 below. First we do the estimate for $H_{0}$ :

Lemma A.2. Let $f$ denote a multiplication operator by a function $f(x) \in$ $L^{2}(\mathbb{R})$. For $\lambda \in \mathbb{C} \backslash[0, \infty), f \cdot\left(\lambda-H_{0}\right)^{-1}$ is Hilbert-Schmidt and its HilbertSchmidt norm satisfies

$$
\left\|f \cdot\left(\lambda-H_{0}\right)^{-1}\right\|_{2} \leq C_{\varepsilon} \cdot\|f\|_{L^{2}} \cdot|\lambda|^{-3 / 4} \quad \text { for } \Re \lambda<\varepsilon|\operatorname{Im} \lambda|-\varepsilon,
$$

given $\varepsilon>0$.

Proof. The Hilbert-Schmidt norm can be expressed in terms of the integral kernel:

$$
\begin{aligned}
\left\|f \cdot\left(\lambda-H_{0}\right)^{-1}\right\|_{2}^{2} & =\int_{\mathbb{R}^{2}}\left|f(x)\left(\lambda-H_{0}\right)^{-1}(x, y)\right|^{2} d x d y \\
& =\int_{\mathbb{R}^{2}}|f(x)|^{2} \frac{1}{2|\lambda|} \cdot e^{-2 \Re \sqrt{-\lambda}|x-y|} d y \\
& =\frac{1}{2}|\lambda|^{-1} \cdot(\Re \sqrt{-\lambda})^{-1} \cdot\|f\|_{L^{2}},
\end{aligned}
$$

which yields the estimate of the Lemma.

Applying the formula of Proposition A.2, we obtain

Proposition A.3. For $f \in L^{2}(0, \infty)$, we have the Hilbert-Schmidt estimate

$$
\left\|f \cdot\left(\lambda-\bar{H}_{\kappa, \alpha}\right)^{-1}\right\|_{2} \leq C_{\varepsilon} \cdot\|f\|_{L^{2}} \cdot|\lambda|^{-3 / 4} \quad \text { for } \Re \lambda<\varepsilon|\Im|-\varepsilon .
$$

Resolvent Products. The derivatives of the heat kernel of $H_{\kappa, \alpha}$ were expressed in terms of the operators

$$
B_{\beta, d, \lambda}=x^{-\beta} \partial_{x}^{d}\left(\lambda-\bar{H}_{\kappa, \alpha}\right)^{-1}
$$


which are defined in Proposition A.1. Given a finite sequence of these operators, $\Pi=\left\{B_{\beta_{j}, d_{j}, \lambda}\right\}_{j=1}^{k}$, we will refer to an operator product of the form

$$
\operatorname{rp}(\Pi)=\prod_{j=1}^{k} B_{\beta_{j}, d_{j}, \lambda}
$$

as a resolvent product.

The operator (A.1) is well-defined if that is the case for each of the factors, i.e., if

$$
1-\left(\frac{\beta_{j}}{\alpha}+\frac{d_{j}}{2}\right) \geq 0, \quad j=1, \ldots, k
$$

The crucial observation is that (A.1) is defined by holomorphic extension to values of the $\beta_{j}$ beyond the domain defined by (A.2).

Proposition A.4. Suppose each $d_{j}$ is fixed to the value 0 or 1 . The operator (A.1) extends to a bounded-operator-valued holomorphic function of $\beta_{1}, \ldots, \beta_{k}$ in the domain

$$
\begin{array}{ll}
\Re \beta_{j} & >0, \quad j=1, \ldots, k \\
\Re i(\Pi) & >0
\end{array}
$$

where $i(\Pi)$ is the decay index of $\Pi$ :

$$
i(\Pi)=k-\sum_{j=1}^{k}\left(\frac{\beta_{j}}{\alpha}+\frac{d_{j}}{2}\right) .
$$

For $\lambda$ sufficiently large in $\Re \lambda<\varepsilon|\Im \lambda|$ (for any given $\varepsilon>0$ ), we have the operator norm estimate

$$
|| r p(\Pi) \| \leq C_{\varepsilon} \cdot|\lambda|^{-\Re i(\Pi)} .
$$

Proof. The result is immediate from Proposition A.1 when $k=1$. For arbitrary $k$, it is reduced to the $k=1$ case in the part of the domain (A.3) where $\Re i(\Pi)>k-1$. In fact, when $\Re i(\Pi)>k-1$, we must have $\frac{\beta_{j}}{\alpha}+\frac{d_{j}}{2}<1$ for all $j$.

We will use double induction to prove the existence of the holomorphic extension with the bound (A.5) in each of the domains

$$
\mathcal{D}_{k, R}=\left\{\begin{array}{ll}
\Re \beta_{j} & >0, \\
\Re i(\Pi) & >R
\end{array} \quad j=1, \ldots, k\right.
$$

where $k=1,2, \ldots$ and $R \geq 0$. We will do this by writing $\operatorname{rp}(\Pi)$ as a combination of resolvent products with fewer factors or of higher decay index. 
The basic step is to commute a factor that contains all the dependence on $\beta_{1}$ to the right of the resolvent $R_{\lambda}=\left(\lambda-\bar{H}_{\kappa, \alpha}\right)^{-1}$ in $B_{\beta_{1}, d_{1}, \lambda}$ :

$$
\begin{aligned}
B_{\beta_{1}, d_{1}, \lambda}= & x^{-\frac{2-d}{2} \alpha} \partial^{d} R_{\lambda} x^{-\left(\beta-\frac{2-d}{2} \alpha\right)} \\
& +x^{-\frac{2-d}{2} \alpha}\left[x^{-\left(\beta-\frac{2-d}{2} \alpha\right)}, \partial^{d}\right] R_{\lambda} \\
& -x^{-\frac{2-d}{2} \alpha} \partial^{d} R_{\lambda}\left[x^{-\left(\beta-\frac{2-d}{2} \alpha\right)}, \partial_{x}^{2}\right] R_{\lambda}
\end{aligned}
$$

The last term on the right comes from the commutator with $R_{\lambda}$; if $\varphi$ is a multiplication operator by a function,

$$
\left[\varphi, R_{\lambda}\right]=R_{\lambda}\left[\varphi, H_{\kappa, \alpha}\right] R_{\lambda}=-R_{\lambda}\left[\varphi, \partial_{x}^{2}\right] R_{\lambda}
$$

We compute the commutators explicitly and find that $\operatorname{rp}(\Pi)$ is a linear combination of the following four operators:

$$
\begin{aligned}
& B \cdot \operatorname{rp}\left(\tilde{\Pi}\left(\beta_{2}+\beta_{1}-\frac{2-d_{1}}{2} \alpha, \beta_{3}, \ldots, \beta_{k}\right)\right) \\
& d_{1} \cdot \operatorname{rp}\left(\Pi_{0}\left(\beta_{1}-1, \beta_{2}, \ldots, \beta_{k}\right)\right) \\
& B \cdot \operatorname{rp}\left(\Pi_{0}\left(\beta_{1}-(\alpha-2)+d_{1} \cdot \frac{\alpha}{2}, \beta_{2}, \ldots, \beta_{k}\right)\right) \\
& B \cdot \operatorname{rp}\left(\Pi_{2}\left(\beta_{1}-(\alpha-1)+d_{1} \cdot \frac{\alpha}{2}, \beta_{2}, \ldots, \beta_{k}\right)\right)
\end{aligned}
$$

where

$$
\begin{aligned}
B & =x^{-\frac{2-d_{1}}{2} \alpha} \partial^{d_{1}} R_{\lambda} \\
\tilde{\Pi}\left(\beta_{2}, \ldots, \beta_{k}\right) & =\left\{B_{\beta_{j}, d_{j}, \lambda}\right\}_{j=2}^{k}, \\
\Pi_{d}\left(\beta_{1}, \ldots, \beta_{k}\right) & =\left\{B_{\beta_{j}, d_{j}, \lambda}\right\}_{j=1}^{k} \quad\left(\text { with } d_{1}=d\right) .
\end{aligned}
$$

The decay indices of the resolvent products in (A.6) are

$$
i\left(\Pi_{0}\left(\beta_{1}+1, \beta_{2}, \ldots, \beta_{k}\right)\right)=i(\Pi)-\frac{1}{\alpha}+\frac{1}{2}
$$


where we set $d_{1}=1$, because this term is only present when $d_{1} \neq 0$,

$$
\begin{aligned}
i\left(\Pi_{0}\left(\beta_{1}-(\alpha-2)+d_{1} \cdot \frac{\alpha}{2}, \beta_{2}, \ldots, \beta_{k}\right)\right) & =i(\Pi)+2\left(\frac{1}{2}-\frac{1}{\alpha}\right) \\
i\left(\Pi_{1}\left(\beta_{1}-(\alpha-2)+d_{1} \cdot \frac{\alpha}{2}, \beta_{2}, \ldots, \beta_{k}\right)\right) & =i(\Pi)+\left(1-\frac{1}{\alpha}\right)-\frac{1}{2} \\
& =i(\Pi)+\left(\frac{1}{2}-\frac{1}{\alpha}\right) .
\end{aligned}
$$

The $\tilde{\Pi}$-term is a resolvent product with $k-1$ factors. For the induction step assume that the result of the proposition holds for resolvent products with fewer than $k$ factors in the subset $\mathcal{D}_{k, k-1-m-\left(\frac{1}{2}-\frac{1}{\alpha}\right)}$ of the domain (A.3) for some integer $m$ such that $k-1-m\left(\frac{1}{2}-\frac{1}{\alpha}\right) \geq 0$. If $\Re i(\Pi)>$ $\max \left(0, k-1-(m+1) \cdot\left(\frac{1}{2}-\frac{1}{\alpha}\right)\right)$, it follows that the conclusion of the proposition still holds, because our decay index calculations above show that all of the operators (A.6) are covered by the induction hypothesis.

\section{References}

[ABP] M. F. Atiyah, R. Bott and V. K. Patodi, On the heat equation and the index theorem, Invent. Math. 19 (1973), 279-330.

[BrS] J. Brüning and R. T. Seeley, Regular singular symptotics, Adv. Math. 58 (1985), 133-148.

[C1] C. J. Callias, The heat equation with singular coefficients, I, Comm. Math. Phys. 88 (1983), 357-385.

[C2] - The resolvent and the heat kernel for some singular boundary problems, Comm. P.D.E. 13 (1988) (9), 1113-1155.

[C3] - The heat kernel as a function of the coefficients of the singularity in the potential, I: a differential calculus of power-log asymptotic expansions, preprint.

[C4] _ The heat kernel as a function of the coefficient of the singularity in the potential, II: the regular singular case, preprint.

[C5] - Spectrally determined singularities in a potential, Comm. P.D.E. (in press).

[C6] Spectrally determined singularities in a potential, II, preprint.

[CT] C. J. Callias and C. H. Taubes, Functional determinants in Euclidean Yang-Mills theory, Comm. Math. Phys. 77 (1980), 229-250.

[CU] C. J. Callias and G. A. Uhlmann, Singular asymptotic approach to partial differential equations with singular coefficients, Bull. AMS. (Research Announcements), July 1984.

[Ch] J. Cheeger, Spectral geometry of singular Riemannian spaces, J. Diff. Geom. 18 (1983), 575-651.

[E2] M. Economakis, Boundary regularity of the harmonic map problem between asymptotically hyperbolic manifolds, Ph.D thesis, University of Washington, Seattle (July 1993).

[Gi] P. Gilkey, Invariance theory, the heat equation, and the Atiyah-Singer index theorem, Publish or Perish, Boston (1984). 
[Gr] P. Greiner, An asymptotic expansion for the heat equation, Proc. Symp. Global Analysis, AMS (1968) 137-146; Arch. Rat. Mech. Anal., 41 (1971), 163-218.

[Gu] V. Guillemin, Spectral theory on $S^{2}$ : some open questions, Adv. Math. 42 (1981), 283-290.

[Ma] R. Mazzeo, Elliptic theory of differential edge operators I, Comm. P.D.E., 16, 1615-1664 (1991).

[Me] R. Melrose, Calculus of conormal distributions on manifolds with corners, International Mathematical Research Notices, No. 3, 51-61 (1992).

[OPS] B. Osgood, R. Phillips and P. Sarnak, Compact isospectral sets of surfaces, J. Funct. Anal. 80 (1988), 212-234.

[RS] M. Reed and B. Simon, Methods of modern mathematical physics, vol. II, Academic Press, New York (1975).

[S] R. T. Seeley, Complex powers of an elliptic operator, Proc. Symp. Pure Math. 10 (1967), 288-307.

Department of Mathematics, University of Crete, Iraklion, Crete, Greece E-mail address: callias@talos.cc.uch.gr 